\title{
SERVICIOS DE SALUd DE LOS ELITES SUCRE A LAS COMUNIDADES EXCLUIDAS
}

\author{
Dilma Diany Hallasi Roselló, John R. Tinco Bautista, Felix A. Medrano Cárdenas, Hugo Infanzón Escobar, Elizabeth \\ Guzmán Vidalón, Lucio Dipaz Saez y José Vega Salas ${ }^{1}$
}

\section{RESUMEN}

La acción de las brigadas de salud de los Equipos Locales Itinerantes de Trabajo Extramural en Salud (ELITES) del Ministerio de Salud (MINSA) con apoyo de la Comunidad Europea, para beneficio de los peruanos de las zonas excluidas, es poco conocida. Este servicio se remonta a 1905. A partir de junio de 1998 se estableció como atención constante. Los pobladores de las comunidades cuando enfermaban no recibían ningún tipo de atención, ahora son atendidos en medicina, odontología, enfermería y obstetricia principalmente. ELITES apoya en la promoción y articulación de los servicios organizando con las autoridades de las comunidades en la formación de los Comités de Desarrollo Comunal (CODECO). También organiza y capacita a los promotores de salud. Los miembros del equipo llegan a las comunidades a pie portando en sus mochilas el equipo básico, los medicamentos y sus efectos personales. Comparten el alimento de los pobladores y duermen en los ambientes que les proporcionan. Las brigadas son buena imagen para el gobierno en el aspecto salud.

Palabras clave: Brigadas, salud, excluidas, desarrollo, comunidades.

\section{ABSTRACT}

The action of the brigades of health of the Traveling Local Equipment of Extramural Work in Health (ELITES) of the Ministry of Salud (MINSA) with support of the European Community, in benefit of the Peruvians from excluded zones, is little well-known. This service goes back to 1905. As of June of 1998 it was settled down like constant attention. The population of the communities when they became ill did not receive any type of attention, now are taken care of in medicine, odontology, nursing and obstetrics mainly. ELITES supports in the promotion and joint of the services organizing with the authorities of the communities in the formation of the Committees of Communal Development (CODECO). Also, it organizes and enables the health promoters. The members of the equipment arrive at the communities on foot carrying in their knapsacks the basic equipment, medicines and their personal effects. They share the food of the population and sleep in the atmospheres that they provide to them. The brigades are good image for the government in the aspect health.

Key words: Brigades, health, excluded, development, communities

\section{INTRODUCCIÓN}

La brigada de ELITES tiene la misión de aliviar el sufrimiento de familias socialmente excluidas con patrones de asentamiento dispersas que viven en forma disgregada por milenios, con el fin que alcancen sus propias potencialidades en armonía con la sociedad de la que forman parte. Gran parte de la población asistida considera que por fin el gobierno se preocupa por ellos.

En el campo de la salud, el $56,7 \%$ de la población en extrema pobreza que enferman, no reciben ningún tipo de atención, en el departamento de Ayacucho hasta 1998 fue aproximadamente de 50000 personas. La falta de atención son atribuidas a los escasos recursos económicos de la población $62 \%$, deseo de no recibir atención $13,8 \%$, problemas de accesibilidad y disponibilidad de servicios de salud $9,4 \%$, otras causas $17,8 \%$.
Además para cada 10000 habitantes se cuenta con 4,3 médicos, lo que es insuficiente en relación a nivel nacional con el 10,3 médicos. Aquí las comunidades dispersas y excluidas que son el $23 \%$ de la población, no recibe ningún tipo de servicio de salud ni de educación, es más limitado a las mujeres; su economía es de autocosumo.

\section{Antecedentes}

Desde que se instaló el servicio de salud en la zona, han ocurrido las siguientes experiencias:

Cuerpo de vacunadores 1905. Fue creado por decreto para la aplicación de la ley sobre el uso de vacunas de enero de 1896, que establecía que los prefectos departamentales debían cuidar que los médicos titulados y sanitarios practicaran "un servicio de vacunación y revacunación extraordinario".

Brigada sanitaria de Manuel Núñez
Butron 1933. El doctor Núñez Butron organizo las brigadas sanitarias en 1933. La primera en Isla comunidad de 31999 habitantes cerca de Juliaca. La brigada recibió el nombre quechua "Nichay" que significa despierto. De los "Nichay" se dice que "no son sanitarios para curar son nicharys para evitar". Se incorporó a curanderos y herbolarios los que sin renunciar a sus creencias aceptaban algunos métodos de Nuñez Butron. Las principales actividades fueron de vacunación y la promoción de la higiene para prevenir el tifus. Atendían partos, purgaban, sacaban muelas, enseñaban a fabricar jabón y construían pequeñas estufas que sin maltratar la ropa mataban los piojos. Convocaban a la población a la plaza del pueblo y explicaban las ventajas de la vacunación y la higiene. Utilizaban canciones populares con letras que promovían la alfabetización y la higiene. Publicó la revista "Runa Soncco" con noticias sanitarias, salieron 10 números entre

\footnotetext{
${ }^{1}$ Miembros del equipo ELITES Sucre, Dirección Regional de Salud de Ayacucho (DIRESA) E-mail: dhallasi@hotmail.com
} 
1935 y $1948(1,2)$.

Experiencia reciente. En junio de 1998 el Programa de Administración de Acuerdos de Gestión (PAAG) inició el plan piloto itinerante en las cuencas de los ríos Ene y Tambo en Junín, y del Alto Bravo y Bajo Huallaga en San Martín. La atención se realizó por las brigadas de ELITES del Ministerio de Salud (MINSA) para brindar atención de salud básica. Fue diseñado para brindar en su mayor parte para recuperación de la salud en las "poblaciones excluidas". Durante el año 2002 el MINSA contó con 50 ELITES en 19 direcciones de salud en la amazonía y 11 en la sierra. Los ELITES han incorporado en la atención itinerante a 220292 personas distribuidas en 1723 poblaciones, 65 comunidades nativas y 1072 centros poblados. El total de atenciones a nivel nacional fue de 721271 .

Experiencia de atención en salud a la población dispersa del departamento de Ayacucho. En octubre de 1998 se implementó el piloto Modelo de Atención en Salud a Poblaciones Dispersas (MASPODIPA) en la Microrred Pomabamba - Ayacucho en los distritos de María Parado de Bellido, Sarhua y Chuschi. Se inició en noviembre de 1998 en las zonas de Chungui y Huancasancos. Fue ampliada en las provincias de La Mar, Parinacochas, Sucre, Lucanas, Huanta, Cangallo y Hunacasancos; en el 2000 en las provincias de Cangallo, Víctor Fajardo y Vilcashuamán (3).

\section{MATERIAL Y MÉTODOS}

La brigada Sucre cuenta con un equipo integrado por una médica, un odontólogo, una obstetríz, un enfermero, un antropólogo o un biólogo y un técnico en enfermería. Se desplazan a las zonas de la serranía y parte de selva de acuerdo a un itinerario visitando las comunidades, la mayoría de las veces a pie, portando sus mochilas con su equipo básico, medicamentos y efectos personales. Duermen en los lugares que la comunidad les asigna, generalmente en un ambiente común y sobre cueros de ganado auquénido, caprino u ovino. Comparten el alimento de los pobladores

Atienden consultas y realizan atenciones, trabajan por aproximadamente 21 días luego preparan el informe correspondiente. Además brindan servicio de organización comunal, apoyando, hasta donde les es posible en el aspecto social, educativo y otros que la comunidad necesita.

\section{RESULTADOS}

Las experiencias han demostrado el avance importante para disminuir las brechas en la prestación de servicios en las poblaciones rurales, mejorando las posibilidades de una intervención sanitaria oportuna. La DIRESA ha logrado consolidar el modelo de atención integral de salud basado en la participación social, desarrollo de recursos humanos, calidad, eficiencia y ética, reduciendo los riesgos. Con ocho equipos en todo el departamento.

La brigada Sucre hace una real de promoción de la salud, prevención de la enfermedad y atención, haciendo que los servicios satisfagan las condiciones de accesibilidad económica, geográfica y cultural para los habitantes, asegurando la disponibilidad y la suficiencia de recursos físicos, humanos y financieros. Respetando las creencias propias de cada comunidad.

Durante el periodo de febrero a septiembre del año 2003, se logro atender 1383 pacientes y 8814 atenciones de los cuales fueron 2186 en medicina, 2045 en enfermería 1678 en obstetricia, 2259 en odontología y 646 por tópico.

Las condiciones inhóspitas, la inaccesibilidad adicionado a las limitaciones socioeconómicas en las comunidades, condiciona a la propensión de diferentes enfermedades, ocupa el primer lugar las del aparato respiratorio $35,21 \%$. En segundo lugar se encuentra las infecto parasitarias $14,63 \%$, en estas dos los más afectados son los niños, por las condiciones climatologías, inmunológicas y las socioeconómicas de la región. Las enfermedades del sistema nervioso $12,3 \%$, enfermedades osteomusculares $11,08 \%$ y del aparato digestivo $10,02 \%$ están en tercer, cuarto y quinto lugar respectivamente, están en relación con la población adulta y en menor proporción en el adulto mayor. En sexto lugar se encuentra las enfermedades del aparato genito urinario $3,5 \%$ siendo más afectados las mujeres en edad fértil. En el servicio de odontología el $2,62 \%$ presentó solo caries dental y el $97.38 \%$ presentó caries dental asociado a otra patología de cavidad oral.

\section{DISCUSIÓN}

Ayacucho es uno de los departamentos andinos más pobres del Perú, que arrastra profundas cicatrices de la violencia y con una creciente violencia social, expresión de la angustia y desesperanza de muchos, de enormes contrastes geográficos que propician la gran dispersión de su población, con ancestrales raíces culturales, la marcada pobreza e inequidad en la distribución de los escasos recursos con sus múltiples manifestaciones, los patrones étnicosculturales de su población, genera fragmentación y dispersión de la población rural con problemas de comunicación y acceso, entre otros factores configuran una ancestral situación de exclusión social. Frente a esta situación se ha constituido una naciente respuesta socio sanitaria, que no aún no logra brindar de manera eficaz a la compleja y heterogénea problemática sanitaria, prevalecen modelos de atención sustentados en prácticas sanitarias tradicionales (4).

EI ELITES realiza sus actividades en comunidades seleccionadas con riesgo sanitario, con visitas periódicas por rutas sanitarias pre establecidas, brindando atención integral de servicios de salud individual y colectiva, priorizando la promoción de la salud familiar y comunal; fortaleciendo la red social que busca la organización y empoderamiento de las comunidades para su desarrollo social sostenible con su participación, y, de organizaciones públicas y privadas que ayudan a identificar y priorizar problemas a través de los CODECOs; se van articulando a las mesas de concertación distritales y articulando los servicios de salud a la comunidad, mediante el trabajo coordinado e integrado con el personal de los Establecimientos de Salud (EESS), agentes comunitarios de salud, autoridades locales y comunales, a través de la Implementación del Sistema de Vigilancia Comunal en Salud $(2,3)$

Desde el inicio de la acción, las brigadas han tratado de ganar la confianza de la población con éxito, aún falta mucho para cubrir todas las zonas y para obtener la confianza absoluta con la medicina moderna sin oponerse a la medicina en la que ellos creen culturalmente. Los habitantes de la zona manifiestan confianza al ver que un grupo de profesionales van hasta 
su lugar de origen para atenderlos, generalmente caminando al igual que ellos por la falta de vías de acceso a la movilidad motorizada. Como todo proceso cultural se acostumbrará a esos pobladores a tratarse con los profesionales de salud con tiempo. Por el momento lo que más desean son medicamentos.

Evidentemente este accionar no cubre las expectativas, pero es una esperanza para ellos al saber que las autoridades de salud se preocupan, enviando profesionales calificados que han obtenido el puesto por concurso, lo que da garantía a la calidad del servicio.

Lo mencionado implica que las activi- dades de ELITES deben ser complementadas con acciones de otros profesionales para lograr la mejora en la calidad de vida integral y sostenible en el tiempo. Falta mucho por hacer. El paso inicial está dado para ir ganando progresivamente la confianza al mantenerlas saludables hasta donde es posible. Entienden que con buena salud y buena educación se puede superar las condiciones ambientales para lograr progreso en su zona de arraigo (5).

El éxito es la unidad del equipo de la brigada convencido de su labor. A veces es un poco difícil que seis personas puedan concordar en algunos aspectos. La brigada Sucre trabaja en equipo superando los inconvenientes.
ELITES Sucre realiza su trabajo en las comunidades con acercamiento de la realidad de salud, en donde cada profesional visualiza los servicios de medicina, odontología, enfermería, obstetricia, biología y en la técnica en enfermería en la jurisdicción de la provincia de Sucre.

En el presente año el ELITES Sucre ha servido de enlace entre la comunidad y los EESS cumpliendo el rol de mejorar el nivel de salud y buscando el desarrollo comunal, como estrategia para la sostenibilidad de los trabajos realizados y emprendidos sugiriendo la continuidad de los ELITES.

\section{REFERENCIAS BIBLIOGRAFICA}

1. Dirección Regional de Salud Ayacucho. Propuesta de trabajo de los equipos de oferta móvil en comunidades excluidas. Ayacucho DIRESA; 2003.

2. DIRESA. Informes consolidados de las brigadas de Ayacucho. Ayacucho: Diresa; 2003.

3. DIRESA. Informe gerencial ELITES Sucre. Ayacucho: Diresa; 2003

4. Hallasi Roselló, Dilma. Minería artesanal repercusiones en la salud. Fope.net 2003; 2 (1): $23-25$. En: http://www.fopecal.org/download/Fope.net02.pdf

5. El Peruano. Más de 26 mil damnificados por la ola de frío en el sur. Viernes 12 de julio 2002. Lima: El Peruano. En: http://www.editoraperu.com.pe/edc/02/07/12/nac1.htm 